\title{
De Proust à Burroughs : transformations de la Recherche chez les Beats
}

\author{
Vinciane Boudonnet \\ Trent University
}

Pour quiconque se souvient du volume abîmé de Dean Moriarty, le gourou de On the Road, l'importance de Marcel Proust pour les auteurs américains de la Beat Generation est manifeste. Pardelà les différences du milieu et du ton, Proust est une figure initiatique pour les Beats. Non seulement une des visées du roman proustien - sa recherche du vrai et son corollaire, le démantèlement des apparences et des bienséances sociales mais aussi certaines de ses techniques deviennent des références, le roman de Proust acquérant rapidement un statut incontournable dans le dialogue intertextuel de part et d'autre de l'Atlantique. En effet, l'influence d'À la recherche du temps 
perdu sur les auteurs américains de la Beat Generation s'exerce aussi bien de façon directe qu'à travers leur intégration de l'œuvre de Jean Genet, dont les écrits fournissent sans doute le premier grand exemple d'une réécriture de la Recherche. Comme Genet, Jack Kerouac et William Burroughs reconnaissent explicitement s'être inspirés du travail monumental de Proust, qui repousse les limites de ce qu'il est possible d'exprimer pour fonder une certaine littérature de la marginalité. Mais cette influence est, comme toujours, ambiguë : dans son passage à travers les relectures de Kerouac et de Burroughs, comme déjà dans ses métamorphoses genétiennes, c'est l'œuvre proustienne elle-même qui est finalement marginalisée par les transgressions de la génération suivante.

Les thèmes principaux du roman proustien sont, bien sûr, le temps et l'amour. D'ailleurs, comme on le sait, c'est la découverte de l'homosexualité des personnages (d'Albertine, du baron de Charlus surtout) qui prépare à la révélation ultime par où le temps se renverse dans la structure narrative du roman. Dans la présente étude, je me pencherai sur la dynamique d'influence et de marginalisation qui préside à la réception de ces thèmes proustiens en considérant tour à tour leur traitement dans Notre-Dame-des-Fleurs et Miracle de la Rose de Genet et, pour le temps en particulier, dans On the Road de Kerouac. Enfin, j'évoquerai brièvement l'intersection de ces thématiques dans le roman de Burroughs, Naked Lunch.

Le processus par lequel les romans de Genet prennent forme, leur génétique si l'on veut, passe par le renversement des mécanismes proustiens. Là où la narration proustienne se bâtit sur la découverte de la sexualité des personnages (de même que sur celle de leur identité à travers le temps) comme 
révélation d'un principe caché, l'homosexualité (et la criminalité avec laquelle elle se confond) est chez Genet le thème exclusif et explicite d'une écriture qui vise plutôt à inverser les valeurs des apparences bourgeoises. Semblablement, Kerouac adopte et adapte les techniques proustiennes pour appréhender le temps, les intervertissant dans la création d'une esthétique de l'instant. Perdant son caractère totalisant de «ruban de Moebius " rétrospectif, le roman proustien persiste comme une référence, un symbole de l'insaisissable dans une recherche pour laquelle l'incomplétude devient la marque d'authenticité. La rencontre des thématiques sexuelle et temporelle dans le roman de Burroughs s'effectue sous des conditions différentes pour ce qui est du rapport au modèle proustien. S'il est difficile d'établir une influence qui présiderait à l'identité des thématiques dans les deux ouvrages, les «méprises» au sens de Bloom (1979) sont au contraire évidentes. En une image à la fois parallèle et inversée des métamorphoses en insectes énormes que subissent les personnages de Naked Lunch, l'ouvrage monumental de Proust devient méconnaissable dans un discours pour lequel l'émancipation du désir ne dépend plus du travail de l'individu, qu'il s'agisse du personnage social ou du «moi profond» du créateur proustien, mais passe au contraire par sa liquidation.

\section{L'inverti de Proust et les personnages gais de Genet}

L'une des facettes de l'œuvre proustienne qui donne à voir le plus clairement cette dynamique d'influence et de marginalisation est celle qui concerne l'homosexualité. Si cette thématique qui structure en grande partie la Recherche occupe les devants de scène chez les auteurs de la génération suivante, 
tous, Genet en tête, refusent la construction de l'homosexualité comme vérité cachée de l'inverti proustien et rejettent en parallèle l'esthétique correspondante de la littérature comme révélation.

Dès la fin des années 1930 et le début des années 1940, la Recherche représente certainement l'influence littéraire la plus importante dans l'œuvre de Genet; cependant, associer Proust et Genet n'est pas à première vue évident: les phrases tentaculaires de Proust, ces «phagocytes capables d'ingérer chaque détail» (White, 1993, p.170) contrastent avec les périodes plus concises de Genet, comme l'emploi généralisé du discours indirect chez Proust tranche avec l'argot des personnages que Genet transcrit directement, donnant ainsi vie aux discours des mondes parallèles et méconnus des prisons, des maisons de correction, du monde de la pègre et de la prostitution dans le Paris de l'époque.

En outre, Genet, qui était lui-même un délinquant, découvrit l'écriture en prison; Proust, quant à lui, écrivait dans le calme de sa chambre tapissée de liège. Au-delà de l'incarcération auto-imposée du neurasthénique et l'incarcération pénale du prisonnier, leur inversion sexuelle les confine tous les deux dans les marges de la société, où chacun recrée un monde à soi. Dans les œuvres de Genet, la sexualité et la mémoire deviennent les sujets extrovertis d'une identité textuelle qui glorifie la marginalité. Le fait que Genet extériorise la rhétorique proustienne de l'inversion transforme l'écriture de l'homosexualité en l'écriture, souvent provocatrice, d'un monde différent et étrange.

Car c'est dans le traitement de l'homosexualité que les différences entre Proust et Genet se font plus évidentes. Chez 
Proust, le narrateur de la Recherche observe patiemment, à distance, la révélation de l'homosexualité; il fait un compte rendu minutieux de la découverte du secret de l'homosexualité seulement révélé du fait de son indiscrétion, le narrateur étant bien sûr exclusivement hétérosexuel. C'est stratégiquement au milieu de son œuvre, au début de Sodome et Gomorrhe, que Proust choisit de mettre au jour la révélation la plus importante de la Recherche : la découverte de l'homosexualité du baron de Charlus. Jusqu'alors, le baron avait toujours été présenté sous des traits virils et paraissait même être un cliché de masculinité et d'hétérosexualité. La révélation de son inversion sexuelle provoque chez Marcel, le narrateur, un moment d'illumination au cours duquel il s'aperçoit que ce qu'il tenait pour acquis n'est autre que le contraire de la vérité. Marcel se trouve être le témoin d'une scène étrange impliquant le baron de Charlus et Jupien, un ancien giletier, et c'est lors de la première rencontre de ces deux hommes, rencontre tout à fait fortuite, que la transformation de Charlus se fait jour :

À ce moment, où il ne se croyait regardé par personne, les paupières baissées contre le soleil, M. de Charlus avait relâché dans son visage cette tension, amorti cette vitalité factice, qu'entretenaient chez lui l'animation de la causerie et la force de la volonté. Pâle comme un marbre, il avait le nez fort, ses traits fins ne recevaient plus d'un regard volontaire une signification différente qui altérât la beauté de leur modelé; plus rien qu'un Guermantes, il semblait déjà sculpté, lui Palamède XV, dans la chapelle de Combray. Mais ces traits généraux de toute une famille prenaient pourtant dans le visage de M. de Charlus une finesse plus spiritualisée, plus douce surtout. Je regrettais pour lui qu'il adultérât habituellement de tant de violences, d'étrangetés déplaisantes, de potinages, de dureté, de susceptibilité et d'arrogance, qu'il cachât sous une brutalité postiche l'aménité, la bonté qu'au moment où il sortait de chez Mme de Villeparisis, je voyais s'étaler si naïvement sur 
son visage. Clignant des yeux contre le soleil, il semblait presque sourire, je trouvai à sa figure vue ainsi au repos et comme au naturel quelque chose de si affectueux, de si désarmé, que je ne pus m'empêcher de penser combien $M$. de Charlus eût été fâché s'il avait pu se savoir regardé; car ce à quoi me faisait penser cet homme qui était si épris, qui se piquait si fort de virilité, à qui tout le monde semblait odieusement efféminé, ce à quoi il me faisait penser tout d'un coup, tant il en avait passagèrement les traits, l'expression, le sourire, c'était à une femme! (Proust, 1988, p. 5-6)

La féminisation de Charlus, l'inversion de ses traits masculins, est mise en valeur par le retardement de la révélation dû à la présence d'énumérations et à la constante évocation des yeux de Charlus, de leur expression et, bien sûr, par la fameuse scène métaphorique parallèle à la rencontre de Charlus et de Jupien : celle de la rencontre d'un bourdon (qui représente le baron) avec une orchidée (qui représente Jupien). Les deux scènes s'imbriquent, révélant ainsi l'inversion :

[...] le baron ayant soudain largement ouvert ses yeux mi-clos, regardait avec une attention extraordinaire l'ancien giletier sur le seuil de sa boutique, cependant que celui-ci, cloué subitement sur place devant M. de Charlus, enraciné comme une plante, contemplait d'un air émerveillé l'embonpoint du baron vieillissant. Mais, chose plus étonnante encore, l'attitude de M. de Charlus ayant changé, celle de Jupien se mit aussitôt, comme selon les lois d'un art secret, en harmonie avec elle. Le baron [...] allait, venait, regardait dans le vague [...]. Or Jupien, perdant aussitôt l'air humble et bon que je lui avais toujours connu, avait - en symétrie parfaite avec le baron - redressé la tête, donnait à sa taille un air avantageux, posait avec une impertinence grotesque son poing sur la hanche, faisait saillir son derrière, prenait des poses avec la coquetterie qu'aurait pu avoir l'orchidée pour le bourdon providentiellement survenu. (Proust, 1988, p. 6) 
Cette scène s'étend sur plusieurs pages et constitue l'un des moments clés de toute la Recherche; c'est à ce moment que la vérité de ce qui était caché apparaît, que l'inversion est révélée. Ici, le personnage le plus masculin s'avère être tout son contraire : un inverti. Notons que Proust insiste pour utiliser le mot inverti pour décrire ses personnages parce que ce mot combine à la fois les notions de renversement et d'homosexualité. Ainsi, on voit que la signification véritable des personnages apparaît après que les conventions sociales ont été bouleversées. Cependant, l'inversion sexuelle, bien que révélée avec surprise au narrateur-personnage principal, apparaît sous les traits de la beauté, cernée de références métaphoriques symboliques : celles des fleurs, de la douceur du regard et des gestes. Voyant la transformation des invertis ainsi dépeinte, le lecteur reconnaît les valeurs symboliques de toute une tradition littéraire, à commencer, pour ce qui est des métaphores florales, par les Métamorphoses d'Ovide.

Comme le remarque Burroughs, Genet emploie à son tour ce style, ces images qui marquent le classicisme de sa prose. Ces techniques sont pourtant employées au service d'un objectif bien éloigné de la satire des mœurs proustienne. Comme chez Baudelaire, elles servent à révéler la beauté de l'abjection; chez Genet, elles servent de surcroît à renverser les valeurs sociales en dévoilant la sainteté des criminels et en sacrant l'infâme. En même temps, et de façon toute proustienne en cela, son projet exige qu'il évite le recours aux images sensationnelles comme aux condamnations banales en faveur d'une analyse détaillée, d'une présentation stylistique hautement travaillée surtout, qui maîtrisent rigoureusement les réactions du lecteur. 
Chez Genet, comme chez Proust, c'est le moment de la rencontre qui est important pour les jeunes couples de prisonniers et, là encore, le regard du lecteur accompagne ceux des personnages alors que la transformation qu'ils subissent bouleverse les lois du monde ordinaire. Dans Miracle de la Rose, le narrateur raconte ainsi sa rencontre dans les escaliers de la prison de Mettray avec le jeune codétenu Bulkaen : « [...] pour se poser sur moi, les yeux de Bulkaen s'adoucirent jusqu'à n'être qu'un rayon de lune frissonnant de feuilles et sa bouche sourit. Les murailles s'effritaient, le temps tombait en poudre, Bulkaen et moi demeurions debout sur une colonne qui nous élevait toujours plus haut.»(p.63) Comme dans Sodome et Gomorrhe, c'est ici la transformation qui opère : transformation du masculin en féminin et aussi, par l'entremise métonymique d'un tatouage, d'espèce : «Il m'y parlait avec admiration des bagarres et des bagarreurs. Et cela après la métamorphose de l'aigle en femme me le fit craindre plus viril qu'il ne le paraissait à son visage. » (Genet, 1946, p. 92)

Comme Proust encore, Genet utilise la symbolique des fleurs. Sans doute guidé dans ce transfert par la réalité de son propre nom, Genet s'identifie à ses personnages et se réfère à eux constamment en termes de fleurs. Mais si Proust fait appel à la signification généralement comprise par la culture dominante en associant les fleurs à la beauté et à la douceur, Genet en renverse les valeurs et en fait des symboles de beauté associés au crime, aux mœurs honnies, renversement que complexifie l'opposition des genres (féminin pour les fleurs et masculin pour les jeunes marles qu'elles caractérisent). Dans un transfert de valeurs qui engage le lecteur et l'auteur au même titre que les personnages et dont l'expérience est une initiation à la sainteté genétienne, les fleurs que l'on trouve dans les noms 
de ses personnages (Notre-Dame-des-Fleurs et Mimosa, par exemple) évoquent un érotisme de beaux meurtriers : " Mettray grouillait d'enfants aux visages, aux corps et aux âmes séduisants. J'ai vécu parmi ce petit monde cruel [...] les durs et leur braguette entrouverte d'où s'échappent par bouffées qui vous soulèvent le cœur le parfum des roses thé et des glycines s'oubliant vers le soir [...]. » (Genet, 1946, p. 75) ${ }^{1}$

L'exemple le plus frappant de cette technique est sans doute la scène où Harcamone, meurtrier condamné à mort qui fascine d'amour et d'admiration le narrateur, apparaît lors de son ultime sortie de cellule; moment d'apothéose où opère le miracle de la rose :

Harcamone laissa tomber les bras et la chaîne pendit devant lui, plus bas que la ceinture. Il sortit de cellule. Comme les tournesols vers le soleil, nos visages se tournèrent et pivotèrent nos corps sans même que nous nous rendissions compte que notre immobilité était dérangée et, quand il s'avança vers nous, à petits pas comme les femmes de 1910 à la robe entravée, ou comme lui-même dansait la java, nous eûmes la tentation de nous agenouiller ou, tout au moins, de poser la main sur nos yeux, par pudeur. [...] Je sentais dans toutes mes veines que le miracle était en marche. Mais la ferveur de notre admiration avec la charge de sainteté qui pesait sur la chaîne serrant ses poignets - ses cheveux eurent le temps de pousser, leurs boucles s'embrouillaient sur son front avec la cruauté savante des torsades de la couronne d'épines - firent cette chaîne se transformer sous nos yeux [...], en une guirlande de roses blanches. [...] j'avançais de deux pas, le corps penché en avant, les ciseaux à la main, et je coupai la plus belle rose qui pendait à une tige souple, tout près de son poignet gauche. (Genet, 1946, p. 26)

\footnotetext{
1 Ainsi que le présente le narrateur de Notre-Dame-des-Fleurs, «Culafroy et Divine aux goûts délicats, seront toujours contraints d'aimer ce qu'ils abhorrent et cela constitue un peu de leur sainteté, car c'est du renoncement. » (Genet, 1948, p. 79)
} 
L'action de couper la fleur évoque l'exécution du jeune meurtrier dans le geste même qui accomplit sa métamorphose en rosier.

Partout présente chez Genet, la mort est violente et même glorieuse lorsqu'il est question de condamnations à la peine capitale. Cela relève en partie de la transfiguration systématique des valeurs qu'il opère: dans le monde qu'il dépeint, l'abjection, la déviance, le crime, le mal prédominent et deviennent normaux, et la mort même devient un événement heureux dans la Colonie de Mettray telle que la recrée l'imaginaire de Genet: "Des souvenirs que j'ai, les plus tristes sont joyeux. Les enterrements furent des fêtes [...]. » (Genet, 1946, p. 243)² En fait, si Genet souhaite créer sa propre légende, exposant les détails d'une sexualité marginale, c'est non seulement dans le but de ne pas oublier sa jeunesse mais aussi pour s'approprier sa propre mort. Quête du passé, d'une enfance perdue, l'écriture est aussi chez Genet comme chez Proust une tentative de transcendance, ou du moins d'appropriation du discours de l'immortalité. À sa façon, Genet veut aussi forcer les portes de l'éternité. Cette recherche du temps est d'ailleurs explicitement rattachée par Genet à sa lecture de Proust ${ }^{3}$.

\footnotetext{
2 Genet a en effet écrit sa vie d'enfant abandonné et constamment réinventée par sa propre légende, qu'il a voulu aussi réelle que mythique. Pour lui, " [t]out ce que nous faisons, nous le faisons pour avoir un bel enterrement, des funérailles cérémonielles. Mes funérailles seront mon chef-d'œuvre, mon œuvre principale, le plus grand accomplissement de toute ma vie. » (1948, p. 210)

3 Dans une entrevue datant de 1983 avec R. Wischenbart et L. S. Barrade, Genet explique: «Créer, c'est parler de son enfance. C'est toujours nostalgique. C'est le cas dans mes écrits et dans la plupart des écrits modernes. Vous connaissez [...] la première phrase de l'œuvre de Proust : "Longtemps je me suis couché de bonne heure." et il raconte son enfance qui
} 
Aussi la transposition des sexes et le renversement des valeurs sociales préparent-ils à leur tour une participation esthétique au topos fondamental du triomphe de la parole vraie sur la mort. Cependant, l'inversion sexuelle n'est pas chez Genet une anormalité exceptionnelle permettant d'entrevoir des vérités ésotériques, mais la seule norme qui soit dans son univers.

Dans une image inversée du grand monde disséqué dans la Recherche, le «Proust du Paris marginal »4 nous submerge dans le spectacle de la déshérence, de vies déconstruites évoluant selon des règles contraires à ce que la société des gens dits normaux accepte. Cette inversion de la perception de la vie lui permet de dépeindre le beau dans le laid, le bien dans le mal, le sacré dans la profanation. Cependant, le renversement en question n'est pas présenté comme le fruit d'une longue recherche, révélé dans un moment d'illumination. À l'opposé des distinctions proustiennes entre le moi profond et le moi social, comme aussi entre le Marcel homo- et hétéro-diégétique, Genet travaille à fusionner sa propre personnalité avec chacun de ses personnages, notamment dans Notre-Dame-des-Fleurs, où il attribue jusqu'au lieu de son enfance à l'un de ses personnages appelé Divine. Et ce procédé est foncièrement lié au désir non seulement d' $d^{\prime}$ assumer » l'érotique masculine mais de s'en servir pour repousser l'opinion bien-pensante au-delà des derniers retranchements de son indulgence libérale. Genet

dure mille cinq cent, plus de deux mille pages en fait. J'avais trente ans quand j'ai commencé à écrire. Et trente-quatre ou trente-cinq quand j'ai arrêté. Mais c'était un rêve, [...] un rêve éveillé. J'ai écrit en prison. Une fois libéré, j'étais perdu. » (1991, p. 277)

4 Je traduis : «[...] Genet is the Proust of marginal Paris. » (White, 1993, p. 169) 
avait tout à fait conscience de ne pas être semblable à son auteur préféré en ce qu'il acceptait totalement les implications «antisociales» de l'homosexualité. Ainsi, dans une entrevue accordée en 1956, il déclare son homosexualité et ajoute :

Un homosexuel est un homme pour lequel le sexe féminin, soit la moitié de l'humanité, n'existe pas. [...] Pour lui, la romance est seulement une sorte de stupidité ou de leurre - pour lui, seul le plaisir existe. Vivre avec surprise, dans le changement, accepter les risques, s'exposer aux insultes : c'est le contraire de la contrainte sociale, de la comédie sociale. De là, si l'homosexuel décide de tenir plus ou moins un rôle dans cette comédie, comme Proust [...], il triche, il ment : tout ce qu'il dit devient suspect. Mon imagination est submergée dans l'abjection mais au moins elle est noble, elle est pure. Je rejette le leurre; et si j'exagère ou si je pousse mes héros ou leurs aventures vers ce qui est effrayant ou abject, ce n'est qu'une exagération dans la direction de la vérité. (Poulet, p. 10-11)

Et c'est conscient $d u$ caractère anormal que possède l'homosexualité dans le monde ordinaire qu'il choisit de transformer ce qui est considéré comme vice en vertu :

Reparler de sainteté à propos de relégation fera crisser vos dents inhabituées aux nourritures acides. Pourtant la vie que je mène requiert ces conditions d'abandon des choses terrestres qu'exigent de ses saints l'Église et toutes les églises. Puis elle ouvre, elle force une porte qui donne sur le merveilleux. Et la sainteté se reconnaît encore en ceci, c'est qu'elle conduit au Ciel par la voie du péché. (Genet, 1946, p. 215)

Dans sa version genétienne, le discours sur l'inversion se retourne contre Proust pour mieux mettre en œuvre la leçon sur le renversement des apparences. Remontant pour s'y ressourcer aux lieux chrétiens transposés par la Recherche sur le plan esthétique, au renversement des valeurs comme au triomphe sur la mort, Genet leur applique les techniques de 
transmutation proustiennes pour forger un style qui ouvre sur une transcendance absolue.

\section{Proust et le temps kérouacien}

Dans cet extrait d'une interview accordée à Miklos Zsedely le 14 avril 1964, Jack Kerouac, questionné sur la littérature, se joue de l'ignorance de la personne qui l'interroge :

JK : Qui a dit : «Un bel après-midi, un dimanche après-midi »? Je crois que c'était Balzac: «Je devais faire la sieste avant de sortir avec mon nouveau cousin passionnant, un garçon, qui venait de rencontrer des filles qu'on appelle des filles de station balnéaire vers les arbres du bord de mer. » Il s'allonge sur son lit pour faire la sieste, mais il ne peut pas dormir et le soleil se couche sur la Manche en Normandie et brille depuis l'Ouest, et cependant depuis la Bretagne, sur sa bibliothèque, de telle sorte qu'il parvient à voir divers paysages, des paysages marins, sur sa bibliothèque. Le niveau supérieur du verre avait un paysage marin, le niveau du milieu, et les livres étaient dedans et tous ces divers paysages marins. Il dit: «Et en contraste à ces énormes paysages marins rose », qui étaient bleus, orange, et jaunes, «je ne pus penser à rien d'autre qu'à la lumière scintillante de la promesse de cette nuit sans sommeil quand je serai avec mon cousin préféré, écoutant les valses mélodieuses des valses viennoises et dansant sur la musique. » Bon sang, ce mec était vraiment nul. Bon, qu'est-ce que vous voulez que je dise à propos de Proust? (Zsedely, p. 236; je traduis.)

À plusieurs reprises au cours de la discussion, Zsedely change de sujet ou tente de recadrer l'interview selon la direction qu'il veut lui faire prendre, ignorant les commentaires de Kerouac alors que ce dernier engage la conversation sur ses auteurs préférés. Dans cet exemple où il semble tester les connaissances de Zsedely en faisant référence au roman de Proust (1987, 
p. 161-163), il termine même sur une note d'humour qui passe totalement inaperçue. Mais en fait, pour celui qui en saisit la subtilité, cette référence va au cœur de l'esthétique kérouacienne, de même que le passage auquel Kerouac se réfère est emblématique de l'esthétique proustienne (voir Boudonnet, 2010).

Le temps perdu, qui est retrouvé chez Proust dans la description même de sa perte, est bien sûr ce qu'évoque ironiquement Kerouac en exprimant combien «nul était ce mec ». En effet, dans la Recherche, le narrateur est insensible à la beauté de ces reflets dans le moment où il en a la sensation, préoccupé qu'il est par l'anticipation du plaisir qu'il prendra à la compagnie des jeunes filles qu'il rencontrera sur sa route. En réalité, l'idéal de la reconquête du temps à travers l'écriture est le moteur même des romans de Kerouac. Comme Seymour Krim le rapporte dans son introduction à Desolation Angels, Kerouac fit, dès 1960, un commentaire concernant les rapports de son œuvre avec celle de Proust : «Mon œuvre comprend un grand livre, comme celui de Proust sauf que mes souvenirs sont écrits à la hâte au lieu de l'être après coup dans un lit de malade... »5. Pareillement, dans une interview accordée en octobre 1962, lorsqu'il est questionné sur sa prose, Kerouac répond : « J'ai lu toute la Recherche du temps perdu de Marcel Proust, et j'ai décidé de faire exactement ce qu'il a fait, mais vite. $»^{6}$

5 « My work comprises one vast book like Proust's except that my remembrances are written on the run instead of afterwards in a sick bed... ». Krim, Desolation Angels, Londres, Panther Books, 1972, p. 19 ; cité dans Dezon-Jones (1982, p. 178).

6 « Now, I read all the Remembrance of Things Past by Marcel Proust, and I decided to do just like he did, but fast. » ("Dialogues in Great Books», entretien accordé à Charles E. Jarvis et James Curtis pour WCAP Radio 
En fait, le bourlingage, dans les romans de Kerouac, pour autant qu'il s'étende dans l'espace, n'est qu'une matière première qui attend d'être ressaisie à travers la remémoration scripturaire. D'ailleurs, l'identité foncière du bourlingueur kérouacien et du voyageur dans le temps est soulignée par le narrateur du roman dans un passage où leur non-identité apparente est attribuée aux défaillances de la mémoire. Lorsque Sal le narrateur et Dean se retrouvent de nouveau à l'est, à New York, Sal évoque une sensation étrange, un souvenir qu'il peine à ressaisir :

Just about that time a strange thing began to haunt me. It was this: I had forgotten something. There was a decision that I was about to make before Dean showed up, and now it was driven clear out of my mind but still hung on the tip of my mind's tongue. I kept snapping my fingers, trying to remember it. [...]. It haunted and flabbergasted me, made me sad. [...]. It had to do with the Shrouded Traveler. [...]. Something, someone, some spirit was pursuing all of us across the desert of life and was bound to catch us before we reached heaven. Naturally, now that I look back on it, this is only death: death will overtake us before heaven. The one thing we yearn for in our living days, that makes us sigh and groan and undergo sweet nauseas of all kind, is the remembrance of some lost bliss that was probably experienced in the womb and can only be reproduced (though we hate to admit it) in death. (Kerouac, 1991, p. 1127)

Interview à Lowell, Massachussetts —ville natale de Kerouac-, reproduit dans Maher, p. 192).

7 C'est exactement à ce moment qu'un sentiment étrange commença à me hanter. C'était ceci : j'avais oublié quelque chose. Il y avait une décision que j'étais sur le point de prendre avant que Dean rapplique et maintenant elle m'était complètement sortie de l'esprit mais elle me restait mentalement sur le bout de la langue. Je ne cessais pas de me mordre les doigts en essayant de m'en souvenir. [...]. Cela me hantais et j'en étais ahuri et triste. Cela avait quelque chose à voir avec le Voyageur au Suaire. [...]. Quelque chose, quelqu'un, quelque esprit devait poursuivre chacun de nous à travers le désert de la vie et il devait de toute nécessité nous saisir avant que nous 
Ici, le recoupement thématique des épisodes de la mémoire involontaire chez Proust est doublé par l'évocation explicite de la remembrance of some lost bliss, le titre anglais du roman de Proust étant bien sûr Remembrance of Things Past, dans la version de Scott Moncrieff, qui reprend le célèbre vers de Shakespeare. Le voyageur enveloppé de mystère n'est autre que le temps, mais qui apparaît ici sous les traits allégoriques de la mort. Au moment où l'esthétique kérouacienne se rapproche le plus de celle de Proust, l'on saisit aussi les différences fondamentales qui l'en séparent. Si les expériences de voyages sont transfigurées par leur remémoration dans les romans de Kerouac, elles demeurent les parties d'un tout qui ne se reconstituent jamais. Aussi l'opération de la métaphore comme figure de l'identité simultanée de moments séparés dans le temps et dans l'espace est-elle toujours subordonnée à la narration d'un prochain voyage.

D'ailleurs, lorsque vient le moment, à la fin du roman, où Proust est explicitement évoqué comme le héros du gourou de la route, Dean Moriarti, il s'avère que la seule réplique à la réminiscence chez les protagonistes kérouaciens est le silence :

[...] suddenly I noticed the hush in the room and looked around and saw a battered book on the radio. I knew it was Dean's high-eternity-in-the-afternoon Proust. As in a dream I saw him tiptoe in from the dark hall in his stocking feet. He couldn't talk any more. [...]. He was listening to sounds in the night. 'Yes!' he

atteignions le paradis. Naturellement, maintenant que je reviens sur cette énigme, il s'agit simplement de la mort: la mort nous rejoindra avant le paradis. La seule chose après laquelle nous languissons durant notre existence, qui nous fait soupirer et gémir et souffrir toutes sortes de doucereuses nausées, c'est le souvenir de quelque félicité perdue que l'on a sans doute éprouvée dans le sein maternel et qui ne saurait se reproduire (mais nous nous refusons à l'admettre) que dans la mort. (trad. Houbart, p. 176-177) 
whispered with awe. 'But you see - no need to talk any more and further.' [...], Sal, I can talk as soon as ever and have many things to say to you in fact with my own little bangtail mind I've been reading and reading this gone Proust all the way across the country and digging a great number of things I'll never have TIME to tell you about and we STILL haven't talked of Mexico and our parting there in fever - but no need to talk. Absolutely, now, yes? (Kerouac, 1991, p. 2788)

Le temps dût-il être définitivement retrouvé, la route le serait aussi, et comme on le voit, récupérer pleinement la mémoire des voyages passés est rendu impossible, justement par une absence de temps face à la nécessité de raconter d'autres voyages. Figures d'une expérience métonymique, les voyages débordent la possibilité de les résorber dans une métaphore totalisante.

Il ne faudrait pas en conclure que les romans de Kerouac ne connaissent pas la fusion de l'être et du temps. Mais cette fusion est elle-même vécue et présentée comme un moment éphémère inscrit à son tour dans un temps circulaire. De plus, à l'encontre de la séparation proustienne du moi profond et du moi social, et de la transfiguration rétroactive de celui-ci par celui-là, les Beats cherchent à fusionner ces deux moi, à

\footnotetext{
8 « [...] soudain je remarquai le silence de la pièce et regardai autour de moi et aperçus un livre dépenaillé sur la radio. Je reconnus le Proust de Dean, destiné à ses hautes-éternités-de-l'après-midi. Comme dans un rêve, je le vis arriver du vestibule obscur sur la pointe des pieds et en chaussettes. Il ne pouvait plus parler. [...]. Il écoutait des bruits dans la nuit. «Oui, murmura-t-il avec une horreur sacrée. Mais, voyez-vous... plus besoin de parler... ni d'en dire plus. [...]. Sal, je sais parler comme jamais et j'ai des tas de choses à te dire du fait que, avec mon propre esprit de poney écourté, j'ai lu et lu ce vieux Proust tout le long du chemin en traversant le pays et savouré un nombre grandiose de choses que je n'aurai jamais le TEMPS de te dire, et nous n'avons pas ENCORE parlé du Mexique et de notre séparation là-bas en pleine fièvre... mais pas besoin de parler. Absolument, eh bien, oui? » (trad. Houbart, p. 432433)
} 
récapituler dans chaque instant la transfiguration proustienne du temps. Dans un passage situé vers la fin du roman, Kerouac résume à merveille le rapport entre l'expérience du temps et la béatitude des Beats :

And for just a moment I had reached the point of ecstasy that I always wanted to reach, which was the complete step across chronological time into timeless shadows, and wonderment in the bleakness of the mortal realm, and the sensation of death kicking at my heels to move on, with a phantom dogging its own heels, and myself hurrying to a plank where all the angels dove off and flew into the holy void of uncreated emptiness [...]. I realized it was because of the stability of the intrinsic Mind that these ripples of birth and death took place, like the action of wind on a sheet of pure, serene, mirror-like water. I felt sweet, swinging bliss, like a big shot of heroin in the mainline vein; like a gulp of wine late in the afternoon and it makes you shudder; my feet tingled. I thought I was going to die the very next moment. (Kerouac, 1991, p. 156-799)

Chez Proust, chaque instant se retrouve à la fin pour faire un tout, une complétude. Chez Kerouac, il n'y a pas ce recentrement du temps; au contraire, tout est disloqué, les moments décrits dans le roman sont tous éparpillés entre le

\footnotetext{
9 Pendant un instant j'avais atteint ce degré d'extase que j'avais toujours convoité, qui était le franchissement total du temps mesurable jusqu'au règne des ombres intemporelles, le ravissement dans le désert de notre condition mortelle, l'impression que la mort me chassait devant elle à coup de pied, ellemême talonnée par un spectre, si bien que je ne trouvais mon salut que sur une planche où les anges, pour y voler, plongeaient dans l'abîme sacré du néant d'avant la création [...]. Je compris que c'était uniquement à cause de la stabilité de l'Esprit essentiel que se produisaient ces fluctuations de naissances et de mort, ainsi le vent ride une nappe d'eau pure et paisible comme un miroir. J'éprouvais une béatitude douce, vacillante, comme si j'avais eu une bonne dose d'héroïne dans la veine; comme après une rasade de vin en fin d'après-midi, et soudain vous frissonnez; j'avais des fourmillements dans les pieds. Je me dis que j'allais mourir dans un instant. (trad. Houbart, p. 243-244)
} 
présent et le passé, comme les personnages. À la fin de On the Road, Sal, qui a rencontré la femme qu'il disait avoir cherchée si longtemps, refuse de repartir sur la route avec Dean. Ce dernier retourne seul vers l'Ouest, à une quête perdue d'avance contre le temps. À la fin du roman, les personnages mêmes qui faisaient l'objet de la remémoration sont de nouveau absents.

En accélérant l'esthétique proustienne pour tenter de faire coïncider temps retrouvé et temps perdu (le moi social et le moi profond) dans une succession de moments vécus plutôt que dans un moment transcendantal qui reste extérieur à l'œuvre pour la fonder, Kerouac inverse les rapports proustiens de la métaphore et de la métonymie pour faire de l'évocation métonymique de leur coïncidence dans la Recherche une figure de l'esthétique vécue des Beats.

\section{"Yage is space-time travel »}

Chez Burroughs, l'extroversion de l'homosexualité et l'explosion de l'unité temporelle se rencontrent pour renverser complètement le modèle de la Recherche et pour fonder une écriture qui rejette en bloc les valeurs littéraires traditionnelles. Toutefois, pour autant qu'il se démarque de cette tradition, Burroughs a toujours affirmé son admiration pour Proust, comme aussi pour Genet. Il associe d'ailleurs les deux écrivains de sorte qu'il semble que les discours de Genet déterminent en partie son appréhension de la Recherche tout comme sa lecture de Proust influe sur sa réception des romans de Genet. Lisant le roman de Proust comme le récit d'un amour homosexuel (il maintient qu'il est évident qu'Albertine est un homme), il précise : «Bien sûr, Genet n'est pas, d'ailleurs il ne prétend pas 
l'être, un innovateur verbal. Il est dans la tradition classique. C'est un écrivain qui, tout en étant dans cette tradition, échappe à la prison des mots. Il a accompli des choses qu'on n'aurait pas cru réalisables avec des mots. » (Odier, p. 44)

Dans Naked Lunch, le temps et l'espace fusionnent et la sensation de leur intégrité, objet de l'esthétique proustienne comme, mutatis mutandis, celle des écrivains de la génération suivante, est à consommer sous forme de drogue dans un monde rempli d'agents secrets qui traquent les homosexuels afin de les soumettre à une "cure». Éclatés sous forme de bribes de conversations, ces champs fournissent la thématique principale du roman, si tant est qu'on puisse encore parler de thèmes ou de "roman »: "And Lee back to sex and pain and time and yagé, bitter Soul Vine of the Amazon... » (Burroughs, 2001, p. 183). Mais si les textes de Burroughs continuent de partager avec la Recherche les thématiques homosexuelle et temporelle, comme celle de l'emprisonnement, il est clair que son écriture se caractérise principalement par ce qui l'en distingue, par le sens de la rupture avec tout ce que représente le modernisme. Là où cette esthétique suppose un lien caché entre les images ou les sons éloignés, Burroughs est postmoderne en cela que sa technique des cut ups s'emploie contre la toile de fond d'une " guerre des images ». La tentative d'aller vers le monde, de l'incorporer en le transformant, de surmonter les contradictions dans la création d'une fiction, est rejetée au profit de la projection. Il ne s'agit pas seulement de rendre explicite, par une sorte d'explosion non médiatisée sur la page, tout ce qui reste implicite dans les textes du modernisme, mais de refuser jusqu'à l'idée de la profondeur, d'un moi ou d'un texte qui pourrait cacher ou même contenir quelque chose d'implicite. Le conduit stylistique principal de cette nouvelle 
exigence est le démantèlement de la métaphore en faveur d'une forme de métonymie qui rend directement les contradictions qui traversent la conscience. L'on peut comprendre cette technique comme le reflet d'une préoccupation vis-à-vis des contradictions inhérentes aux mécanismes du contrôle social plutôt qu'avec celles qui rivent l'individu, celui-ci étant compris désormais comme une fonction de celles-là. Dans ce sens, Burroughs partage avec Beckett une lecture de Proust qui voit dans son œuvre l'ablation du désir plutôt que son expression littéraire.

Les romans de Genet, Kerouac et Burroughs poursuivent tous la recherche proustienne d'une authenticité travestie par les conventions sociales, et leurs explorations des thématiques homosexuelle, temporelle et carcérale continuent d'élargir les frontières du dicible en subvertissant les fictions primaires de la littérature et de la société. Cependant, à mesure que le marginal se déplace, perdant sa charge de révélation pour venir occuper le centre de leurs œuvres, les héritiers de Proust dessinent un horizon littéraire sans frontières, dépourvu de limites restrictives comme de bornes à transgresser. Finalement, dans le paysage de Burroughs, ne restent à saper que les techniques qui avaient servi à effectuer ces transformations. En rejetant les conventions d'une narration unifiée (en enlevant Marcel de l'opération de la mémoire involontaire), Burroughs rend hommage à Proust comme un éclaireur de l'esthétique beat et, en même temps, il le marginalise. 


\section{Bibliographie}

BL0om, Harold. (1979 [1973]), The Anxiety of Influence: a Theory of Poetry, New York, Oxford University Press.

Boudonnet, Vinciane. (2010), «Échos de la Recherche chez les Beats : Proust sur la route », dans Proust, l'étranger, CRIN 54, Rodopi, Amsterdam-New York, p. 119-129.

BurroughS, William. (2001 [1959]), Naked Lunch, The Restored Text, New York, Grove Press.

DEZon-Jones, Elyane. (1982), Proust et l'Amérique: La fiction américaine à la recherche du Temps Perdu, Paris, A.G. Nizet.

GEnet, Jean. (1946), Miracle de la Rose, Paris, Gallimard, coll. « Folio ».

—. (1948), Notre-Dame-des-Fleurs, Paris, Gallimard, coll. « Folio ».

- (1991), L'Ennemi déclaré : textes et entretiens, dans OEuvres complètes, t. IV, Albert Dichy (éd.), Paris, Gallimard.

Kerouac, Jack. (1991 [1955]), On the Road, London, Penguin Classics.

—. (1960), Sur la route, trad. Houbard, Paris, Gallimard.

-. (1995 [1965]), Desolation Angels, New York, Riverhead Books.

MAHER, Paul. (2005), Empty Phantoms, Interviews and Encounters with Jack Kerouac, New York, Thunder's Mouth Press. 
ODIER, Daniel. (1969), Entretiens avec William S. Burroughs, Paris, Pierre Belfond.

PoulET, Robert. (1956), "Jean Genet: Fouillez l'ordure», Bulletin de Paris, no 145, p. 10-11.

Proust, Marcel. (1987), À l'ombre des jeunes filles en fleurs, dans À la recherche du temps perdu, t. II, Paris, Gallimard, coll. «Bibliothèque de la Pléiade».

—. (1988), Sodome et Gomorrhe et La Prisonnière, dans À la recherche du temps perdu, t. III, Paris, Gallimard, coll. «Bibliothèque de la Pléiade ».

WHITE, Edmund. (1993), Genet, a biography, New York, Vintage Books.

ZSEDELY, Miklos. (2005), « Northport Public Library Oral History Project (April 14, 1964)», in Paul Jr. Maher (éd.), Empty Phantoms: Interviews and Encounters with Jack Kerouac, New York, Thunder's Mouth Press.

\section{Résumé}

Le roman de Proust influence les écrivains de la génération Beat, à la fois directement et par sa réfraction à travers les romans de Genet. Moteur des révélations de la Recherche, l'inversion et la marginalité deviennent chez Genet la matière explicite d'une écriture qui continue cependant de viser la transmutation de l'expérience. Chez Kerouac, la transfiguration rétrospective du temps se transforme en une technique visant une appréhension immédiate de l'expérience. Enfin, les thématiques homosexuelle et temporelle se combinent chez Burroughs dans un éclatement des techniques proustiennes qui marginalise la Recherche en même temps qu'il en prolonge la quête. 


\begin{abstract}
Proust's novel influenced the writers of the Beat Generation both directly and by means of its refraction through the novels of Genet. From hidden side and motor of the Remembrance's revelations, homosexuality and marginality become with Genet the explicit material of texts that nevertheless continue the quest to transmute experience. With Kerouac, the retrospective transfiguration of time is transformed into a technique for the immediate apprehension of experience. Finally, temporal and homosexual thematics combine with Burroughs in an explosion of proustian techniques that both marginalizes and perpetuates the Remembrance of Things Past.
\end{abstract}

